\title{
The Interception Problem of a Celestial Body with the Use of the Collinear Libration Points
}

\author{
Alexander Shmyrov and Dzmitry Shymanchuk \\ Applied Mathematics and Control Processes \\ Saint-Petersburg State University \\ Universitetskii prospect 35, Petergof \\ Saint-Petersburg, Russia, 198504 \\ Emails: a.shmyrov@ spbu.ru,d.shimanchuk@spbu.ru
}

\author{
Leonid Sokolov \\ Mathematics and Mechanics \\ Saint-Petersburg State University \\ Universitetskii prospect 28, Petergof \\ Saint-Petersburg, Russia, 198504 \\ Email: 1.sokolov@spbu.ru
}

\begin{abstract}
This paper studies the problem of construct intercept trajectory when using of the neighborhood of the collinear libration point $L_{1}$ of the Sun-Earth system. A celestial body motion is considered in a rotating frame within the Hill's problem of the circular restricted three-body problem. It is known that collinear libration points are unstable but its instability can be used as a positive factor for maneuvering a celestial body in the near-Earth space. Development methodology of control algorithms for the orbital motion of a celestial body when using the instability of collinear libration points is offer. The construct intercept trajectory is based on the properties of a specially introduced phase variables function, called "hazard function". The results of the numerical integration are graphically illustrated.
\end{abstract}

\section{INTRODUCTION}

Currently known and recorded cases of natural celestial bodies fall to the surface of the Earth. This largely determines the problem of asteroid and comet hazard. Within the framework of solutions to this problem are carried out astronomical observations and monitoring of near-Earth space. As a result, a cataloging of such objects is conducted, their potential threat to the Earth at the international level is assessed, projects to counter this threat are being developed [1].

To solve this problem explores the idea of impact on a potentially dangerous celestial body to alter its trajectory and thus prevent the threat of collision. To achieve this goal in [2] proposed to use gravity assist in near-Earth space. It uses properties of unstable trajectory collision. In our paper, we propose the use of another type of instability, the instability property of the collinear libration points [3].

The theoretical properties of the collinear libration points related to many projects as the NASA and the ESA space activities and protect the Earth from potentially dangerous space objects are used. Since the libration point $L_{1}$ and $L_{2}$ are unstable [4], the questions of retention or stabilization of the motion of the celestial bodies in the neighborhood of these points are relevant [5]-[10]. In some cases, instability may be a positive factor that helps reduce energy costs while maneuvering [3], [11]. These maneuvers may occur on a sufficiently large interval of time by gravity, and other disturbing factors.

To intercept maneuvers in near-Earth space in [3] the developed scheme maneuvering is described. This scheme involves the selection and calculation of the orbit of expectations, and the construction of the active part of the trajectory.

The motion of the celestial bodies in near-Earth space is described by a mathematical model of a special circular restricted three-body problem [5].

The paper presents the results of research and numerical construction of the set of trajectories to intercept potentially dangerous celestial body in the near-Earth space.

\section{EQuations of Control Motion}

The equations of control motion of a celestial body in a rotating reference frame $\mathrm{Ox}_{1} x_{2} x_{3}$ using Hill's problem for solar potential have the form

$$
\left\{\begin{array}{l}
\dot{x}_{1}=x_{2}+y_{1} \\
\dot{x}_{2}=-x_{1}+y_{2} \\
\dot{x}_{3}=y_{3} \\
\dot{y}_{1}=-\frac{3 x_{1}}{\|\mathbf{x}\|^{3}}+2 x_{1}+y_{2}+u_{1} \\
\dot{y}_{2}=-\frac{3 x_{2}}{\|\mathbf{x}\|^{3}}-x_{2}-y_{1}+u_{2} \\
\dot{y}_{3}=-\frac{3 x_{3}}{\|\mathbf{x}\|^{3}}-x_{3}
\end{array}\right.
$$

where $\mathbf{x}=\left(x_{1} ; x_{2} ; x_{3}\right)$ is coordinate vector of the celestial body and $\mathbf{y}=\left(y_{1} ; y_{2} ; y_{3}\right)$ is an impulse vector [5]. The center of mass of the Earth coincides with the origin of the coordinate system, while the $O x_{1}$ axis is directed along the axis connecting the centers of mass of the Earth and the Sun. $\|\cdot\|$ is the Euclidean norm of a vector. In the considered model the units of time and distance were chosen in such a way that the unit of distance is approximately equal to $1.5 \times 10^{6} \mathrm{~km}$ and the unit of time is 58.0916 days (the year divided by $2 \pi$ ). The libration point $L_{1}$ in the rotating coordinate system are stationary and have coordinates

$$
\mathbf{x}^{*}=(1 ; 0 ; 0), \mathbf{y}^{*}=(0 ; 1 ; 0) .
$$

Here, the unit of speed in the adopted model is 298.057 $\mathrm{m} / \mathrm{s} \approx 9.94211 \times 10^{-7}$ the speed of light in a vacuum. The unit of acceleration is equal to $5.93844 \times 10^{-5} \mathrm{~m} / \mathrm{s}^{2} \approx 6.05552 \times$ $10^{-6} \times g$, where $g$ is the standard acceleration of free fall for the Earth. 
Uncontrollable system (1) when $u_{1}=u_{2}=0$ is a Hamiltonian system, where Hamiltonian function $H(\mathbf{x} ; \mathbf{y})$ is represented by the formula

$$
H(\mathbf{x} ; \mathbf{y})=\frac{1}{2}\|\mathbf{y}\|^{2}-\frac{3}{\|\mathbf{x}\|}-\frac{3}{2} x_{1}^{2}+\frac{1}{2}\|\mathbf{x}\|^{2}+x_{2} y_{1}-x_{1} y_{2} .
$$

Note also that the uncontrolled system (1) has no first integrals except the energy integral $H(\mathbf{x} ; \mathbf{y})=$ const. Generally the energy integral is written in the form

$$
-2 H(\mathbf{x} ; \mathbf{y})=C
$$

where $C$ is called constant Jacobi.

To select a control action, we need properties of linear approximation of the system of equations (1) in the neighborhood of $L_{1}$.

Linearized equations of control motion in the neighborhood of collinear libration point $L_{1}$ can be represented as

$$
\left\{\begin{array}{l}
\dot{x}_{1}=x_{2}+y_{1}, \\
\dot{x}_{2}=-x_{1}+y_{2}, \\
\dot{x}_{3}=y_{3} \\
\dot{y}_{1}=8\left(x_{1}-1\right)+\left(y_{2}-1\right)+u_{1} \\
\dot{y}_{2}=-4 x_{2}-y_{1}+u_{2} \\
\dot{y}_{3}=-4 x_{3} .
\end{array}\right.
$$

The matrix of linearized system (2) has the following set of eigenvalues:

$$
\begin{aligned}
& \lambda_{1,2}= \pm \sqrt{1+2 \sqrt{7}}= \pm l \\
& \lambda_{3,4}= \pm i \sqrt{2 \sqrt{7}-1}= \pm i \omega_{\mathbf{e}} \\
& \lambda_{5,6}= \pm 2 i= \pm i \omega_{\mathbf{n}} .
\end{aligned}
$$

The positive value of $\lambda_{1}$ entails instability of collinear libration point $L_{1}$.

The eigenvector of the matrix of system (2) corresponding to eigenvalue $\lambda_{1}$ has the form

$$
\mathbf{b}_{1}=\left(b_{1}^{1} ; b_{1}^{2} ; 0 ; b_{1}^{3} ; b_{1}^{4} ; 0\right)=\left(l^{2}+5 ; \frac{l^{2}-3}{l} ; 0 ; \frac{l^{2}+3}{l} ; 2 ; 0\right) .
$$

Let us denote

$$
d_{1}(\mathbf{x} ; \mathbf{y})=\mathbf{b}_{1} \mathbf{z}
$$

where $\mathbf{z}=\left(x_{1}-1 ; x_{2} ; x_{3} ; y_{1} ; y_{2}-1 ; y_{3}\right)$ is a vector-column.

In equations (2), there is an invariant 5-dimensional manifold filled with bounded trajectories asymptotically approaching two-frequency oscillations with frequencies $\omega_{e}$ and $\omega_{n}$. This variety is defined by the equation

$$
d_{1}(\mathbf{x} ; \mathbf{y})=0
$$

where the function $d_{1}(\mathbf{x} ; \mathbf{y})$ is called "hazard function".

\section{COnstruct CONTROL LAW}

Let a celestial body be moving along the parking orbit, the condition $d_{1}(\mathbf{x} ; \mathbf{y}) \approx 0$ is satisfied. In view of linearized system of equations of motion (2), the behavior of "hazard function" $d_{1}(\mathbf{x} ; \mathbf{y})$ on the trajectories can be represented as

$$
\left.\dot{d}_{1}(\mathbf{x} ; \mathbf{y})\right|_{(2)}=l d_{1}(\mathbf{x} ; \mathbf{y})+b_{1}^{3} u_{1}+b_{1}^{4} u_{2} .
$$

In the parking orbit, the value of the "hazard function" is very little. To ensure speedy departure from the neighborhood of libration point $L_{1}$, the value of $\dot{d}_{1}(\mathbf{x} ; \mathbf{y})$ needs to be changed optimally. We can do this with a small impact. This impact we model by a pulse controls $u_{1}$ and $u_{2}$. Equality (3) provides a mathematical basis for constructing algorithms for stabilization the celestial bodies in the neighborhood of the collinear libration points and maneuvering in near-Earth space using a collinear libration points.

Let the vector of a control action $\left(u_{1} ; u_{2}\right)$ be subject to restriction $u_{1}^{2}+u_{2}^{2}=u^{2}$. Then in order to secure the highest increment in the "hazard function", the vector $\left(u_{1} ; u_{2}\right)$ should be collinear to the vector $\left(b_{1}^{3} ; b_{1}^{4}\right)$. From here we get

$$
\left\{\begin{array}{l}
u_{1}= \pm u \frac{b_{1}^{3}}{\sqrt{\left(b_{1}^{3}\right)^{2}+\left(b_{1}^{4}\right)^{2}}}, \\
u_{2}= \pm u \frac{b_{1}^{4}}{\sqrt{\left(b_{1}^{3}\right)^{2}+\left(b_{1}^{4}\right)^{2}}} .
\end{array}\right.
$$

Here is an example.

\section{EXAMPLE OF INTERRUPTION TRAJECTORIES}

Let a celestial body is in the neighborhood of the parking orbit in the phase space. The calculation of such an orbit has been represented in [3]. We present the results of numerical construction of the set of trajectories to intercept when the control action $u$ approximately $10^{-9} \mathrm{~g}$.

Graphs of the control components shown in Fig. 1. Control operates on the time interval of about 14 hours.

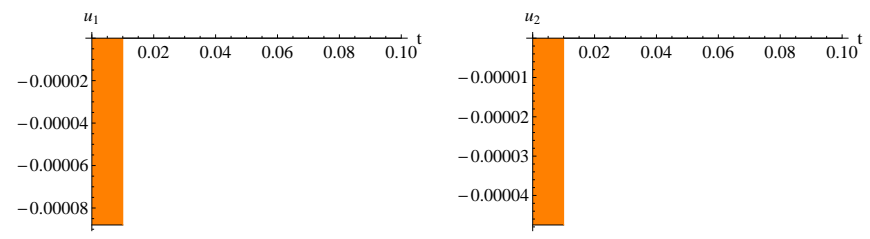

Fig. 1. Control actions $u_{1}$ and $u_{2}(T=0.01)$

Fig. 2 shows the results of numerical integration of the system (1), where the trajectories of a celestial body with the uncontrolled motion (left) and the active control (right). Trajectories are correspond to the time interval of about 6 months.

Fig. 3 shows the dependence of the thrust $\tau$ of the mass $m$ of a celestial body when the control action is approximately equal to $10^{-8} \mathrm{~m} / \mathrm{s}^{2}$. It is noted that the order of thrust is estimated as $10^{-5} \mathrm{~N}$ when the order of mass of a celestial body is several tonnes. 

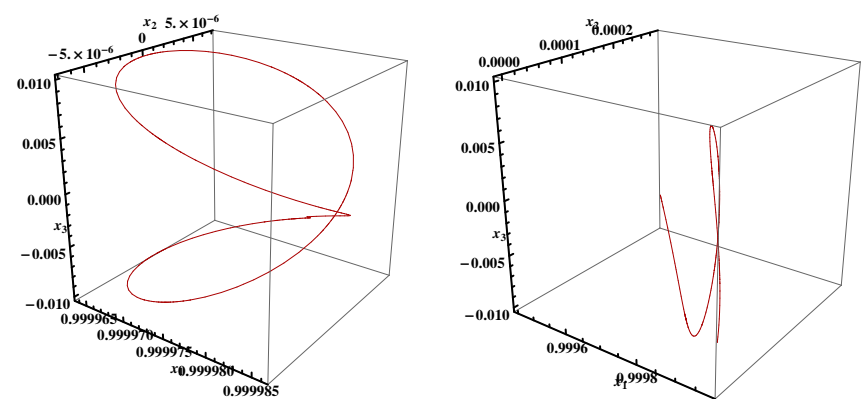

Fig. 2. Motion of the celestial body $(T=\pi)$

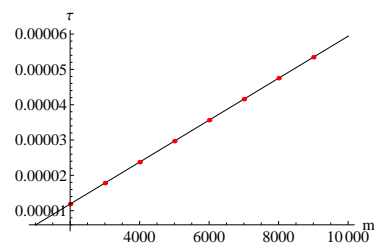

Fig. 3. The dependence of the thrust $\tau(\mathrm{N})$ of the mass $m(\mathrm{~kg})$ of a celestial body

Fig. 4 shows how to use a very small control action $u=$ $10^{-8} \mathrm{~m} / \mathrm{s}^{2}$ celestial body performs a maneuver that provides a sufficiently rapid departure from the neighborhood of a parking orbit of the collinear libration point $L_{1}$. Trajectories of the corresponding period of time of about 14 months. Fig. 4 shows the behavior of the trajectories depending on the celestial body position in the parking orbit of a collinear libration point $L_{1}$.

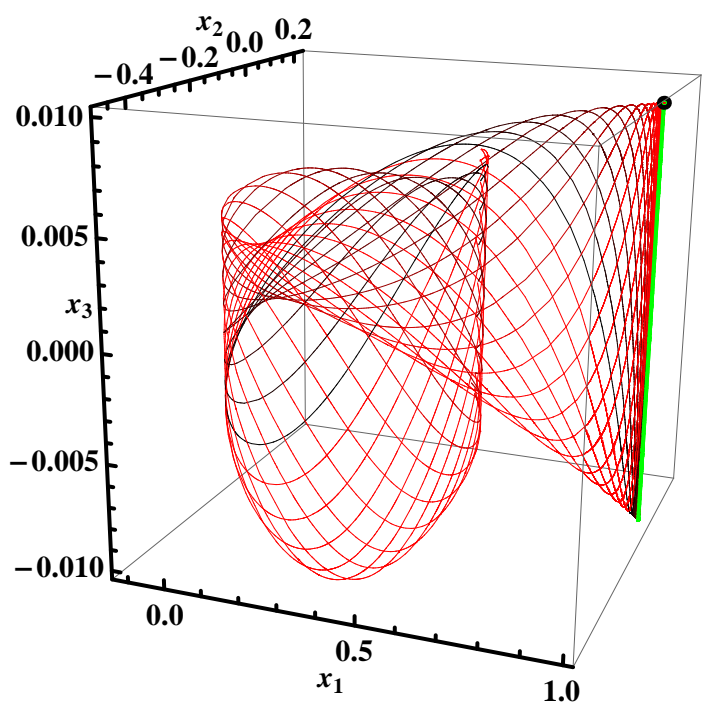

Fig. 4. The set of the intercepting trajectories $(T=7)$

\section{CONCLUSION}

This study dedicated to the orbital motion of a celestial body shows that a small control action can secure sufficiently rapid departure from the neighborhood of collinear libration point $L_{1}$. The example of orbital motion considered show the theoretical possibility of maneuvering a celestial body in the near-Earth space by using the neighborhoods of collinear libration points $L_{1}$. In these cases, instability is achieved by the use of sufficiently small control impacts and thus allows to significantly alter the trajectory of the celestial body at minimal energy costs. Ideally, the energy costs for removal of the managed object from an unstable state can be arbitrarily small. Thus, this makes us an attractive proposition scheme maneuvering to control the orbital motion of celestial bodies with a large mass. These bodies can be used, in particular for impact on potentially dangerous asteroids.

Note also that in [3] have shown that the instability of a collinear libration point has a significant impact on the behavior of plane variables $x_{1}, x_{2}, y_{1}, y_{2}$ and has virtually no effect on the changes in spatial variables $x_{3}, y_{3}$. For $x_{3}$ characteristic oscillations with an amplitude close to the amplitude of the parking orbit.

\section{ACKNOWLEDGMENT}

The authors acknowledge Saint-Petersburg State University for a research grant 9.37.345.2015

\section{REFERENCES}

[1] L. Sokolov, G. Kuteeva, On the consequences of resonant returns of near-earth asteroids, 2015 International Conference on Mechanics Seventh Polyakhov's Reading, art. no. 7106780, 2015.

[2] N. A. Eismont et al., On the possibility of the guidance of small asteroids to dangerous celestial bodies using the gravity-assist maneuver, Solar System Research, vol. 47, no. 4, pp. 325-333. 2013.

[3] A. Shmyrov, D. Shymanchuk, Maneuvering in near-Earth space with the use of the collinear libration points, 2015 International Conference on Mechanics - Seventh Polyakhov's Reading, art. no. 7106777, 2015.

[4] A. P. Markeev, Libration points in celestial mechanics and cosmodynamics, Moscow, Nauka. (in Russian) 1978.

[5] A. Shmyrov, V. Shmyrov, Controllable orbital motion in a neighborhood of collinear libration point, Applied Mathematical Sciences, vol. 8 (9-12), pp. 487-492. 2014.

[6] A. Shmyrov, V. Shmyrov, Method of Lyapunov functions for controllable Hamiltonian systems, 2014 20th International Workshop on Beam Dynamics and Optimization, BDO 2014, art. no. 6890078, 2014.

[7] A. Shmyrov, V. Shmyrov, The estimation of controllability area in the problem of controllable movement in a neighborhood of collinear libration point, 2015 International Conference on Mechanics - Seventh Polyakhov's Reading, art. no. 7106776, 2015.

[8] F. M. Kulakov, A. S. Shmyrov, D. V. Shymanchuk, Supervisory remote control of space robot in an unstable libration point, Proceedings of the 2013 IEEE 7th International Conference on Intelligent Data Acquisition and Advanced Computing Systems, IDAACS, vol. 2, pp. 925-928, 2013.

[9] A. Shmyrov, V. Shmyrov, D. Shymanchuk, Prospects for the use of space robots in the neighborhood of the libration points, Applied Mathematical Sciences, (49-52), pp. 2465-2471, 2014.

[10] G. Maliavkin, A. Shmyrov, Inter-orbital transfer to the neighborhood of a collinear libration point, 2015 International Conference on Mechanics - Seventh Polyakhov's Reading, art. no. 7106752, 2015.

[11] D. V. Shymanchuk, A.S. Shmyrov, Construction of trajectory of the return in the neighborhood of the collinear libration point of the SunEarth system, Bulletin of St.-Petersburg University. Series 10. Release 2. pp. 76-85. (in Russian) 2013. 